\title{
Echocardiographic evaluation of cardiac dyssynchrony for predicting a favourable response to cardiac resynchronisation therapy
}

\author{
C M Yu, J J Bax, M Monaghan, P Nihoyannopoulos
}

Heart 2004;90(Suppl VI):vi17-vi22. doi: 10.1136/hrt.2004.048322

Cardiac resynchronisation therapy (CRT) is an established therapy for patients with heart failure with wide QRS duration. Recent studies observed that assessment of systolic dyssynchrony is an important diagnostic tool as the treatment involves the re-coordination of regional wall contraction within the left ventricle. Therefore, the effectiveness of CRT depends heavily on whether systolic dyssynchrony is present before the treatment. Echocardiography is a useful tool for quantitative measurement of the severity of dyssynchrony in these patients before and after CRT. A number of echocardiographic tools have been developed during the past three years for such purpose, include $M$ mode measurement of septal-to-posterior wall delay, tissue Doppler imaging for septal-to-lateral wall delay, the measurement of standard deviation of peak contraction time over 12 left ventricular segments, delayed longitudinal contraction, and potentially three dimensional echocardiography. This review discusses the potential role of various echocardiographic techniques in the assessment of systolic dyssynchrony and their clinical applications.

$\mathrm{L}$ eft ventricular (LV) dyssynchrony is a relatively common problem in heart failure patients, in particular those with - prolonged QRS complex duration. ${ }^{1}$ Dyssynchronous LV contraction leads to reduced ejection volume because blood moves around the left ventricle from early activated segments to late activated segments. This obviously leads to reduced efficiency in LV contraction and reduces systolic function despite the same energy use. In addition, mitral valve dysfunction frequently occurs because of papillary muscle incoordination. Furthermore, diastolic filling will be in severe jeopardy in the presence of first degree atrioventricular block, especially diastolic dysfunction commonly coexists in systolic failure.

Cardiac resynchronisation therapy (CRT) is a rapidly evolving treatment modality for heart failure. This therapy is characterised by the implantation of the LV lead to the free wall region to pace the left ventricle. The transvenous approach of delivering the LV lead to the lateral or posterolateral cardiac vein through the coronary sinus is the most common way. ${ }^{2}$ This therapy is recommended to patients with advanced heart failure, already on optimal medical treatment, LV ejection fraction $<35 \%$, who had electromechanical delay which is manifested by the prolonged QRS duration on the surface ECG. Clinical trials have confirmed the benefits of CRT on symptoms, exercise capacity, quality of life, and long term prognosis including mortality and heart failure hospitalisation. ${ }^{2-5}$
Echocardiography has an eminent role in the era of CRT. The clinical applications including the assessment of change in systolic function and haemodynamics (for example, LV ejection fraction, cardiac output, $+\mathrm{dp} / \mathrm{dt}$ and myocardial performance index), reduction of LV volume or reverse remodelling, mitral regurgitation and diastolic filling time. ${ }^{6}$ Other than this role, the assessment of cardiac synchronicity by echocardiography is particular important. In general, cardiac dyssynchrony (or asynchrony) is commonly divided into two types: interventricular (between left and right ventricle) and intraventricular (within the LV) dyssynchrony. A number of echocardiographic techniques have been employed by various researchers to assess the coordination of the heart during systole. These include conventional methods such as $M$ mode and Doppler echocardiography as well as newer technologies such as tissue Doppler imaging (TDI), strain rate imaging, and three dimensional echocardiography. This review will discuss the potential role of various echocardiographic techniques in the assessment of systolic dyssynchrony and potential clinical applications.

\section{MODE AND DOPPLER ECHOCARDIOGRAPHY}

$M$ mode echocardiography is the standard feature in every echocardiographic machine. It can be employed to evaluate systolic wall excursion motion in two regions simultaneously. Early work by Xiao and colleagues ${ }^{7}$ examined patients with cardiomyopathy and different degree of QRS prolongation. Patients with classic left bundle branch block and intraventricular conduction delay were delayed in the onset of LV free wall contraction. ${ }^{7}$ The work by Pitzalis and colleagues ${ }^{8}$ examined 20 patients with predominately non-ischaemic aetiology of heart failure who underwent CRT. The echocardiographic examination was performed before and one month after device implantation. Responders to therapy were defined as those exhibiting a reduction of LV end systolic volume index of $\geqslant 15 \%$. The authors reported that $M$ mode assessment of septal-to-posterior wall motion delay of $\geqslant 130 \mathrm{~ms}$ had a specificity of $63 \%$ and positive predictive value of $80 \%$ for LV reverse remodelling.

Pulse Doppler echocardiography can be used to assess the timing of onset of blood flow across the chamber or vessel. When the sampling cursor is placed at the outflow tracts, Doppler echocardiography is a potentially useful tool to assess the time difference in onset of ejection phase between the left and right ventricle. It is one way of assessing interventricular dyssynchrony. This criterion has been used in CARE-HF (cardiac resynchronisation in heart failure) as diagnostic criteria for systolic dyssynchrony to select patients

Abbreviations: CARE-HF, cardiac resynchronisation in heart failure; $\mathrm{CRT}$, cardiac resynchronisation therapy; LV, left ventricular; RV, right ventricular; TDI, tissue Doppler imaging; Ts, time to peak myocardial contraction during the ejection phase 
for CRT.9 However, whether assessment of interventricular dyssynchrony is an effective tool to predict a positive response to CRT remains to be determined.

\section{TISSUE DOPPLER IMAGING}

Tissue Doppler imaging (TDI) allows the measurement of peak systolic velocity of different regions of the myocardium. Moreover, precise timing of peak systolic velocity is possible when the TDI tracings are related to the electrical activity (QRS complex). Integration of this information allows accurate assessment of electromechanical coupling, and evaluation of inter- and intraventricular dyssynchrony.

Interventricular dyssynchrony was evaluated by Rouleau and colleagues ${ }^{10}$; the authors evaluated 35 patients with dilated cardiomyopathy and TDI. An excellent agreement between the QRS duration and interventricular dyssynchrony assessed by TDI was demonstrated. TDI has also been used to assess LV dyssynchrony. The various methods that have been used will be discussed subsequently. The initial studies used pulsed wave TDI. The major drawback from this approach is that sampling is only possible at one position during the cardiac cycle. When different myocardial positions need to be compared (as is necessary to assess dyssynchrony within the LV), multiple acquisitions are required, and the obtained information is thus influenced by heart rate differences, changes in loading conditions, and breathing. In addition, collection of sufficient data information covering multiple myocardial segments is time consuming.

\section{ASSESSMENT OF INTRAVENTRICULAR DYSSYNCHRONY BY TWO SEGMENTAL MODELS}

The study by Garrigue and colleagues ${ }^{11}$ used pulsed wave TDI obtained in two segments (basal septal, basal lateral) and demonstrated that LV dyssynchrony could even be detected in patients with right bundle branch block, which could be reversed after CRT. Interestingly, only those who had accompanied intraventricular dyssynchrony showed the benefits, but not those without. ${ }^{11}$

More recent studies have acquired colour coded TDI data; the main advantages of this approach are that offline analysis is possible and, more importantly, that simultaneous sampling at multiple sites during the same cardiac cycle is possible. Bax and colleagues ${ }^{12}$ have initially evaluated 22 patients with heart failure, dilated cardiomyopathy, and depressed LV ejection fraction $(\leqslant 35 \%)$ with colour coded TDI before CRT. Immediately after CRT, LV ejection fraction improved from 21 (7)\% to 31 (9)\% ( $p<0.05 v$ baseline). This immediate benefit in systolic function was followed by a significant improvement in New York Heart Association (NYHA) functional class, quality of life score, and six minute hall walk distance at three months follow up. LV dyssynchrony was measured by placing two sample volumes on the basal parts of the septum and lateral wall (referred to as the septal-to-lateral delay). Before CRT, the mean septal-tolateral delay was 110 (33) ms, which was reduced to 29 (22) ms immediately after onset of CRT $(\mathrm{p}<0.05 v$ baseline). Of interest, when the pacemaker was switched off, the septal-to-lateral delay returned to baseline values (105 (47) ms, NS $v$ baseline), associated with an immediate drop in systolic function ( $\mathrm{LV}$ ejection fraction returned to $20(6) \%$, NS $v$ baseline). ${ }^{12}$

In a subsequent study, Bax and colleagues ${ }^{13}$ evaluated 25 patients with colour coded TDI (two segment model) before CRT. The patients were identified according to the established selection criteria for CRT: NYHA class III or IV, LV ejection fraction $\leqslant 35 \%$, QRS with left bundle branch block configuration and duration $>120 \mathrm{~ms}$. In the entire group of patients, LV ejection fraction improved from $22(5) \%$ to 31 $(10) \%(\mathrm{p}<0.05)$ after CRT. The septal-to-lateral delay decreased from 71 (38) ms to 36 (34) ms $(\mathrm{p}<0.01)$ after CRT, and the reduction in septal-to-lateral delay was directly related to the change in LV ejection fraction after CRT (fig 1). Interestingly, only responders of systolic function showed the improvement of clinical status which include NYHA class, quality of life and six minute hall-walk distance. Subsequent multivariate analysis demonstrated that the septal-to-lateral delay at baseline was the only predictor of improvement in LV ejection fraction after CRT. Importantly, $76 \%$ of patients with an improvement in LV ejection fraction after CRT had a septal-to-lateral delay at baseline $\geqslant 60 \mathrm{~ms}$, as compared to only $12.5 \%$ of patients without an improvement in LV ejection fraction after CRT $(\mathrm{p}<0.05) .{ }^{13}$

An acute study was conducted by Yu and colleagues ${ }^{14}$ to examine the early benefits of CRT on LV size, function, and intraventricular dyssynchrony. Thirty three patients who underwent CRT were examined by echocardiography before discharge and within 72 hours after device implantation. The device was randomised to biventricular, right ventricular, and no pacing modes. Colour coded TDI was performed and the septal-to-lateral delay was examined. There was reduction of LV volume (end systolic volume: 146 (77) v 122 (69), $\mathrm{p}=0.003)$, increase in LV ejection fraction $(24(12) \% v 30$ $(14) \%, p=0.02$ ), and reduction of mitral regurgitation only during biventricular pacing mode, but not right ventricular (RV) pacing. TDI showed that the septal-to-lateral delay was abolished by biventricular pacing. Interestingly, RV pacing restored systolic dyssynchrony by further delaying the lateral wall contraction. ${ }^{14}$

\section{ASSESSMENT OF INTRAVENTRICULAR DYSSYNCHRONY BY MULTIPLE SEGMENTAL MODELS}

Assessment of multiple segments by TDI has the theoretical advantage of possessing the capability of examining different patterns of systolic dyssynchrony, such as those with delay other than the septal-to-lateral wall. Of course, the time to analyse regional contraction is considerably longer, especially when pulse TDI was employed. This problem is partially overcome by the use of colour coded TDI where cine loops of multiple views are collected and off-line analyses could be performed at multiple segments simultaneous in each orthogonal view.

Ansalone and colleagues ${ }^{15}$ performed pulsed wave TDI in patients with heart failure before CRT. TDI samples were obtained at five positions, and LV dyssynchrony was calculated. The authors demonstrated that LV resynchronisation after CRT was followed by reverse remodelling and improvement in NYHA class and six minute walking distance. Importantly, the magnitude of benefit was related to the LV dyssynchrony as assessed before CRT.

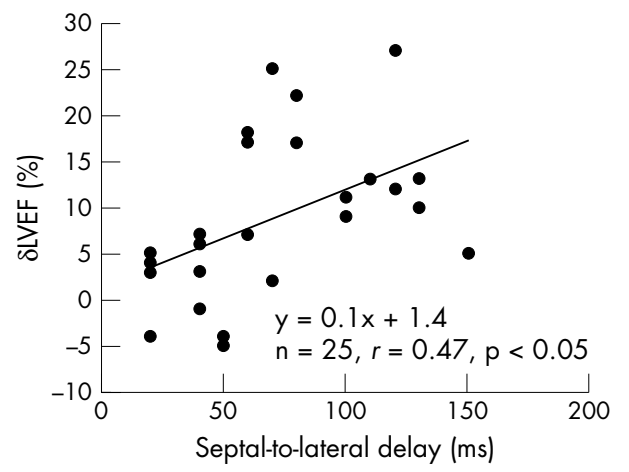

Figure 1 Relation between the left ventricular dyssynchrony at baseline (expressed as septal-to-lateral delay) and the change in left ventricular (LV) ejection fraction after cardiac resynchronisation therapy (CRT). 

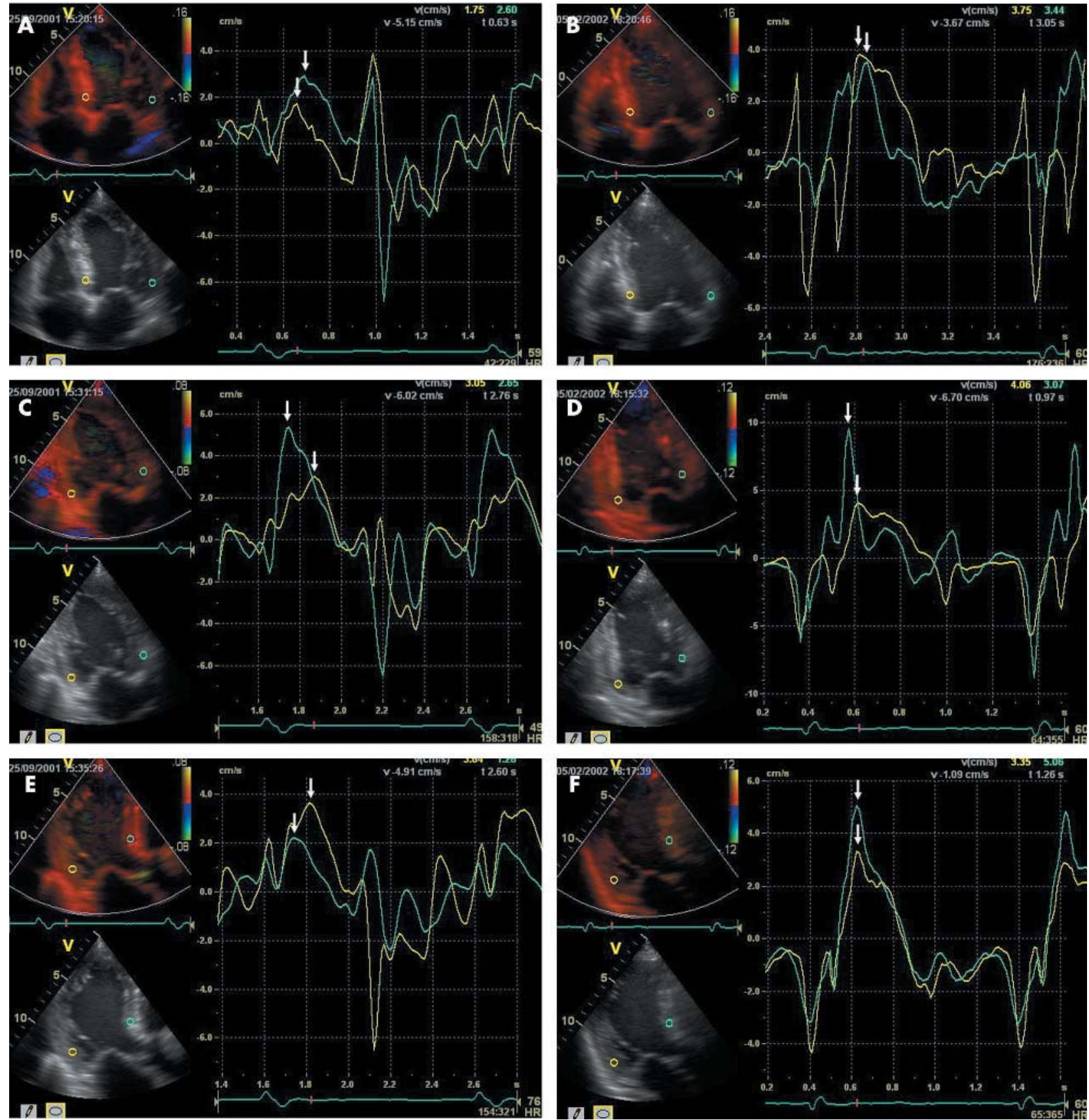

Figure 2 A patient with left ventricular mechanical dyssynchrony in multiple segments before (panels A, C, E) and after (panels B, D, F) CRT. Before CRT, the apical four chamber view (A) shows only mild delay of basal lateral segment over the basal septal segment of $30 \mathrm{~ms}$. In the apical two chamber view (C), there was severe delay in the basal inferior wall over the basal anterior wall of $130 \mathrm{~ms}$ which was significantly improved after CRT (D). In the apical long axis view (E), the basal posterior wall was delayed over the basal anteroseptal wall of 90 ms which was totally abolished after CRT (F). The peak systolic velocity during the ejection phase in each view are shown by the arrows.

The mechanisms on how CRT benefit patients with wide QRS heart failure were examined by $\mathrm{Yu}$ and colleagues ${ }^{16}$ on a mechanical perspective. In that study, 25 patients who underwent CRT were studied sequentially for three months by echocardiography with TDI. The pacing was then withheld for a month in which the same parameters were re-examined. Measurement of time to peak myocardial contraction during the ejection phase (Ts) was performed by TDI at $12 \mathrm{LV}$ segments ( six at the base and six at the middle) by three orthogonal apical views, and the two right ventricular segments. Widespread LV delay was observed among various LV segments, with a large variation in regional Ts. TDI achieved systolic synchronicity by homogenously delaying those early contracting segments to a timing similar to the delayed segments (figs 2 and 3). Therefore, not only was septal-to-lateral delay abolished, other patterns of delay were also corrected. ${ }^{16}$ Furthermore, the septal-to-RV free wall delay was also abolished after CRT. Echocardiographic improvement such as LV reverse remodelling, improvement of systolic function, reduction of mitral regurgitation, and gain in LV diastolic filling time were all pacing dependent, as withholding pacing resulted in progressive worsening of these indices. ${ }^{16}$

Subsequently, $\mathrm{Yu}$ and colleagues ${ }^{17}$ examined for the potential predictor(s) of LV reverse remodelling in 30 patients 


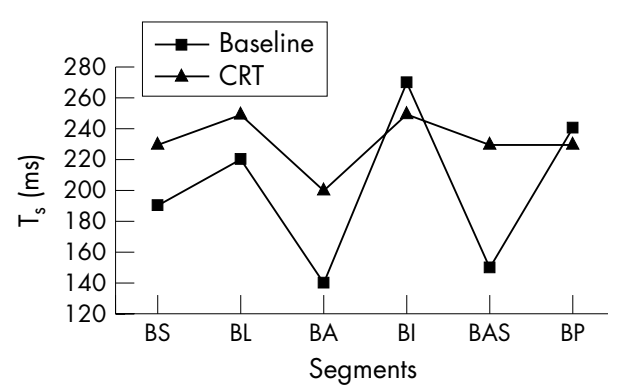

Figure 3 The same patient in fig 2 showing the actual measurement of time to peak systolic velocity during the ejection phase (Ts) of all the six basal left ventricular segments before and after CRT. Before CRT there is a large variation of Ts in the basal segments. After CRT the Ts is homogenously delayed so that the contraction becomes delayed but synchronised in the left ventricle.

who had undergone CRT. The lack of LV reverse remodelling was defined as a reduction of LV end systolic volume of $\leqslant 15 \%$, which was observed in $43 \%$ of patients. In addition, improvement of NYHA class was observed in all the responders of LV reverse remodelling, but only in 54\% of nonresponders. Improvement of peak oxygen uptake, maximal metabolic equivalent achieved during treadmill exercise test, and six minute hall-walk test distance were only observed in the responders. In that study, "asynchrony index" was calculated by calculating the standard deviation of Ts of the $12 \mathrm{LV}$ segments. A larger value indicates more severe LV dyssynchrony. The asynchrony index was observed to correlate with the degree of LV reverse remodelling closely, and was able to predict responders (and non-responders) accurately. In the multivariate model that consisted of clinical, demographic, and echocardiographic parameters, the asynchrony index was the only independent parameter that predicts LV reverse remodelling response. ${ }^{17} \mathrm{~A}$ cut off value of Ts-SD of $32.6 \mathrm{~ms}$, which was derived from a large number of normal control population, was able to segregate responders from non-responders of reverse remodelling. ${ }^{17}$

A recent report by Penicka and colleagues compared the onset of mechanical shortening with ECG signal by pulse Doppler TDI at basal septal, basal lateral and basal posterior segments in the LV, and basal right ventricular free wall segment by apical views. ${ }^{18}$ This method measured the maximal electromechanical delay among the three LV segments as intraventricular dyssynchrony, and compared these three sites with the right ventricular segment for interventricular dyssynchrony. The result observed that adding intra- and interventricular dyssynchrony has a high predictive value to predict responders of CRT who are defined as a relative increase in ejection fraction $>25 \%$. A cut off value of $102 \mathrm{~ms}$ derived from the study population reported an accuracy of $88 \%{ }^{18}$

\section{DELAYED LONGITUDINAL CONTRACTION}

Delayed longitudinal contraction, better known as postsystolic shortening, occurs in a few disease conditions of the heart including myocardial ischaemia, stunning, or hibernation. Recently, it was also found to be a potentially useful surrogate marker of mechanical dyssynchrony in heart failure patients with wide QRS complexes. ${ }^{19}$ The study by Sogaard observed that delayed longitudinal contraction was present in the basal LV segments in these patients, which was reduced from $18.7(7) \%$ at discharge to $8.1(8) \%$ at one year follow up $(p<0.05) .{ }^{19}$ In these 20 patients who received CRT, the improvement of LV ejection fraction correlated with the number of segments with baseline delayed longitudinal contraction.

\section{COMPARISON OF MULTIPLE PARAMETERS OF SYSTOLIC DYSSYNCHRONY TO PREDICT A FAVOURABLE RESPONSE TO CRT}

The list of potentially useful echocardiographic parameters of systolic dyssynchrony is ever increasing. To date, published data on these parameters are highly variable in methodology, ranging from measuring 2-12 parameters in the LV, and compared against different echocardiographic end points, ranging from LV volume to absolute or relative change in ejection fraction. A study by $\mathrm{Yu}$ and colleagues attempted to address this problem by comparing 18 echocardiographic parameters derived from TDI or strain rate imaging for predicting LV reverse remodelling three months after CRT. ${ }^{20}$ In these 56 patients, Ts-SD was confirmed to be the best predictor of LV reverse remodelling, with the greatest correlation coefficient $(r=-0.74, \mathrm{p}<0.001)$ and area of receiver operating characteristics curve (area $=0.94$, $\mathrm{p}<0.001) .{ }^{20}$ The study also observed that models which assessed more segments are more powerful than those that assessed a smaller number of segments; parameters of strain rate imaging are not useful to predict reverse remodelling response; delayed longitudinal contraction is only useful for non-ischaemic aetiology of heart failure; and interventricular dyssynchrony did not predict a favourable response to CRT. ${ }^{20}$

\section{ROLE OF REAL TIME 3D ECHOGRAPHAPHY FOR ASSESSING PATIENTS FOR CRT}

Three dimensional echocardiography provides a unique and powerful tool for the evaluation of LV dyssynchrony (fig 4). The whole ventricular volume dataset is captured over four cardiac cycles with a typical acquisition time of six seconds. Any conventional two dimensional echocardiographic view can be recreated from this dataset and this can be performed on the scanner itself. The dataset can also be transferred to a workstation for off-line analysis.

Off-line analysis allows a more comprehensive and sophisticated evaluation of LV dyssynchrony. The process involves a semi-automated border detection in multiple planes and this avoids manual tracing of the endocardium. The 16 segments defined by the American Society of Echocardiography are identified and a moving ventricular cast displaying all segments is created with visual effect. The software determines the centre of gravity of the left ventricle and the volume of each segment relative to that centre of gravity is calculated. This calculation is performed for every frame in the cardiac cycle so that it is possible to achieve a plot of the volume of every segment against time. Segments should achieve a minimum volume at the same point in the cardiac cycle. However, when there is significant dyssynchrony, the minimum volume time points have wide dispersion. It is possible to use the data derived from the time to minimum volume in each segments to derive a number of useful parameters. These include a systolic dyssynchrony index which may prove to be a powerful predictor of patients who will and will not benefit from CRT.

The advantages of real time three dimensional echocardiography used in this way are that it allows a comparison of synchrony between all segments in the left ventricle together. $\mathrm{M}$ mode and two dimensional echo provide limited intersegment comparison, while tissue Doppler imaging may not be able to compare some of the segments in the same cardiac cycle. The three dimensional echo method provides an intuitive display of ventricular synchronicity which appears to be attractive to non-echocardiographers, in particular electrophysiologists. LV dyssynchrony can be quantified and the systolic dyssynchrony index calculated. As previously stated, this may provide a good prediction of patients who will respond to CRT. It has been shown that patients who had evidence of minimal dyssynchrony are unlikely to get benefit, 
Pre-CRT

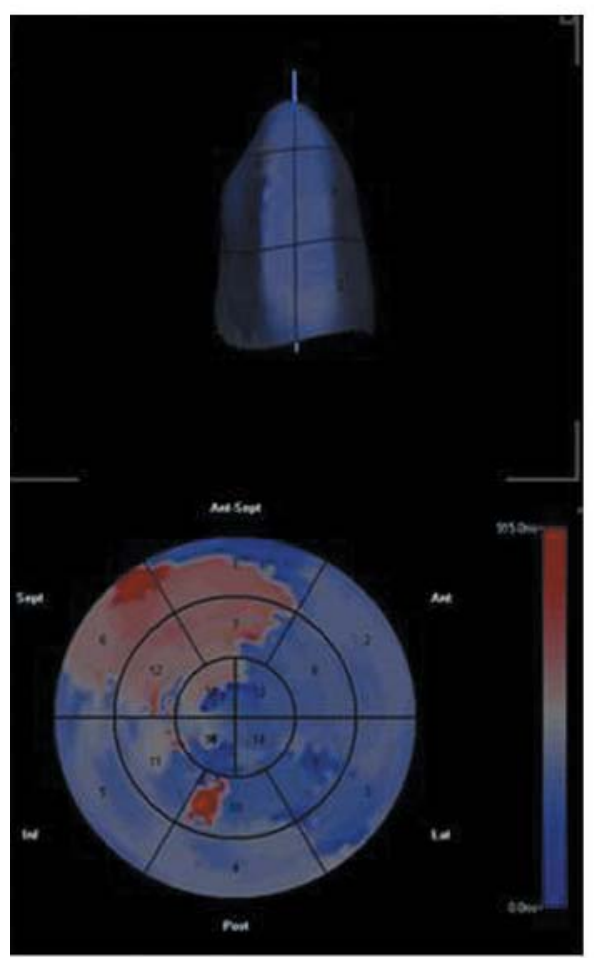

Post-CRT

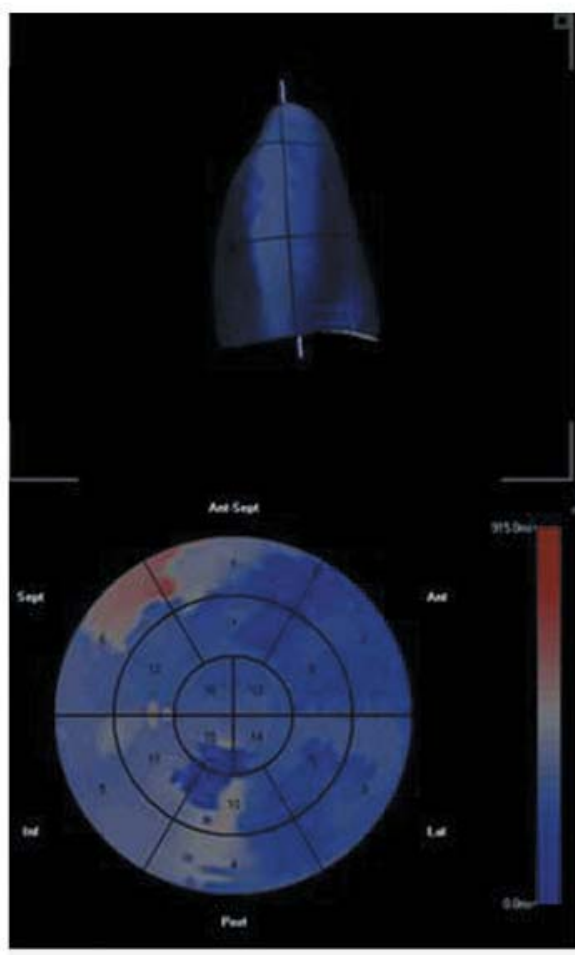

Figure 4 Parametric analysis of three dimensional LV datasets before and after resynchronisation therapy. At the top of each image is an LV cast generated from semi-automated border detection of the LV cavity. Regional contraction is calculated at multiple points over the entire LV endocardial surface and time to maximum contraction is colour coded and displayed on the lower polar maps. Regions with the most delayed contraction are displayed in red and can be seen in the septum before CRT. Following CRT, a significant improvement in the synchronicity of septal contraction is seen in that this area is now mainly coloured blue, as is the rest of the ventricle. irrespective of overall LV function and QRS duration. ${ }^{8} 1317$ However, significant LV dyssynchrony can exist in patients with normal QRS duration and these patients, who would not be eligible for CRT under current criteria, may well benefit from this treatment. ${ }^{21}{ }^{22}$ The technique may also be used to optimise pacemaker settings; a parametric image may be generated in which a polar display of all segments is colour coded according to the timing of achievement of minimal volume. This provides an easy to appreciate, intuitive, and spatially orientated display of dyssynchrony which allows analysis of improvements following CRT and also may help guide optimal placement of pacing electrodes. Finally, image acquisition is relatively rapid compared to other techniques. Clinical data are pending to address the efficacy of three dimensional echocardiography in assessing systolic dyssynchrony and predicting a favourable response.

However, the use of real time three dimensional echocardiography is not without disadvantages. The equipment is relatively expensive and staff need to be trained to use it. The technique can be difficult to use in patients with poor image quality, however, there is good evidence that contrast agents help in this context. The frame rate for wide sector image acquisition is currently low (between 20-30 frame/s), which might affect the accuracy of measuring time domain of the cardiac cycle. While the first generation software for analysis of the datasets was time consuming to learn and to perform, more recent versions have made this a more rapid process.

\section{CONCLUSION}

Clinical results on the assessment of systolic dyssynchrony by various echocardiographic tools are accumulating. It appears clear now that a reproducible method of assessing systolic dyssynchrony is potentially useful if the parameter also predicts a favourable response to CRT. Conventional echocardiographic tools are simple and quick, though may be limited by the lack of ability to assess complex patterns of systolic dyssynchrony in fine details. Newer tools, such as TDI, appear promising and are becoming readily available in all the high end echocardiographic machines. Different algorithms of assessing systolic dyssynchrony by TDI have been proposed, and further studies are needed to compare the predictive value of different parameters in these patients objectively. Delayed longitudinal contraction appears useful although confirmation by other centres is needed. Moreover, the semi-quantitative nature of the method may limit its clinical application, and a unifying definition is needed. New imaging modalities, such as three dimensional echocardiography, are under active development. Its role in CRT is rapidly emerging, though further data are needed to support its clinical utility.

\section{Authors' affiliations}

C M Yu, Division of Cardiology, Department of Medicine and Therapeutics, Prince of Wales Hospital, The Chinese University of Hong Kong, Hong Kong

J J Bax, Leiden University Medical Center, Leiden, The Netherlands M Monaghan, Department of Cardiology, King's College Hospital, London, UK

P Nihoyannopoulos, Imperial College, NHLI, Hammersmith, Hospital, London, UK

Declaration: All authors have read and approved the manuscript and there are no competing interests.

Correspondence to: Professor Cheuk-Man Yu, Division of Cardiology, Department of Medicine and Therapeutics, Prince of Wales Hospital, The Chinese University of Hong Kong, Shatin, NT, Hong Kong; cmyu@cuhk. edu.hk

\section{REFERENCES}

1 Yu CM, Lin H, Zhang Q, et al. High prevalence of left ventricular systolic and diastolic asynchrony in patients with congestive heart failure and normal QRS duration. Heart 2003;89:54-60. 
2 Cazeau S, Leclercq C, Lavergne T, et al. Effects of multisite biventricular pacing in patients with heart failure and intraventricular conduction delay. N Engl J Med 2001;344:873-80

3 Abraham WT, Fisher WG, Smith AL, et al. Cardiac resynchronization in chronic heart failure. N Engl J Med 2002;346:1845-53.

4 Gras D, Leclercq C, Tang AS, et al. Cardiac resynchronization therapy in advanced heart failure-the multicenter InSync clinical study. Eur J Heart Fail 2002;4:31 1-20.

5 Bristow MR, Saxon LA, Boehmer J, et al. Cardiac-resynchronization therapy with or without an implantable defibrillator in advanced chronic heart failure. N Engl J Med 2004;350:2140-50.

6 St John Sutton MG, Plappert T, Abraham WT, et al. Effect of cardiac resynchronization therapy on left ventricular size and function in chronic heart failure. Circulation 2003;107:1985-90.

7 Xiao HB, Roy C, Gibson DG. Nature of ventricular activation in patients with dilated cardiomyopathy: evidence for bilateral bundle branch block. Br Heart J 1994;72:167-74.

8 Pitzalis MV, lacoviello M, Romito R, et al. Cardiac resynchronization therapy tailored by echocardiographic evaluation of ventricular asynchrony. J Am Coll Cardiol 2002;40:1615-22.

9 Cleland JG, Daubert JC, Erdmann E, et al. The CARE-HF study (cardiac resynchronisation in heart failure study): rationale, design and end-points. Eur J Heart Fail 2001;3:481-9.

10 Rouleau F, Merheb M, Geffroy S, et al. Echocardiographic assessment of the interventricular delay of activation and correlation to the QRS width in dilated cardiomyopathy. Pacing Clin Electrophysiol $2001 ; 24: 1500-6$

11 Garrigue S, Reuter S, Labeque JN, et al. Usefulness of biventricular pacing in patients with congestive heart failure and right bundle branch block. Am J Cardiol 2001;88:1436-41.

12 Bax JJ, Molhoek SG, van Erven L, et al. Usefulness of myocardial tissue Doppler echocardiography to evaluate left ventricular dyssynchrony before and after biventricular pacing in patients with idiopathic dilated cardiomyopathy. Am J Cardiol 2003;91:94-7.

13 Bax JJ, Marwick TH, Molhoek SG, et al. Left ventricular dyssynchrony predicts benefit of cardiac resynchronization therapy in patients with end-stage heart failure before pacemaker implantation. Am J Cardiol 2003;92: 1238-40.

14 Yu CM, Lin H, Fung WH, et al. Comparison of acute changes in left ventricular volume, systolic and diastolic functions, and intraventricular synchronicity after biventricular and right ventricular pacing for heart failure. Am Heart J 2003;145:e23-G1-7.

15 Ansalone G, Giannantoni P, Ricci R, et al. Doppler myocardial imaging in patients with heart failure receiving biventricular pacing treatment. Am Heart $J$ 2001;142:881-96.

16 Yu CM, Chau E, Sanderson JE, et al. Tissue Doppler echocardiographic evidence of reverse remodelling and improved synchronicity by simultaneously delaying regional contraction after biventricular pacing therapy in heart failure. Circulation 2002;105:438-45.

17 Yu CM, Fung WH, Lin $\mathrm{H}$, et al. Predictors of left ventricular reverse remodelling after cardiac resynchronization therapy for heart failure secondary to idiopathic dilated or ischemic cardiomyopathy. Am J Cardiol 2003;91:684-8.

18 Penicka M, Bartunek J, de Bruyne B, et al. Improvement of left ventricular function after cardiac resynchronization therapy is predicted by tissue Doppler imaging echocardiography. Circulation 2004; 109:978-83.

19 Sogaard P, Egeblad H, Kim WY, et al. Tissue Doppler imaging predicts improved systolic performance and reversed left ventricular remodelling during long-term cardiac resynchronization therapy. J Am Coll Cardio 2002:40:723-30.

20 Yu CM, Fung JW, Zhang Q, et al. Tissue Doppler imaging is superior to strain rate imaging and postsystolic shortening on the prediction of reverse remodelling in both ischemic and nonischemic heart failure after cardiac resynchronization therapy. Circulation 2004;110:66-73.

21 Achilli A, Sassara M, Ficili S, et al. Long-term effectiveness of cardiac resynchronization therapy in patients with refractory heart failure and "narrow" QRS. J Am Coll Cardiol 2003;42:2117-24.

22 Turner MS, Bleasdale RA, Vinereanu D, et al. Electrical and mechanical components of dyssynchrony in heart failure patients with normal QRS duration and left bundle-branch block: impact of left and biventricular pacing. Circulation 2004; 109:2544-9. 